\title{
Obesidad, tejido adiposo y cirugía bariátrica
}

\author{
María E. Frigolet ${ }^{1}$, Kim Dong-Hoon ${ }^{2}$, Samuel Canizales-Quinteros ${ }^{3,4}$ y Ruth Gutiérrez-Aguilar1,5* \\ ${ }^{1}$ Laboratorio de Enfermedades Metabólicas, Hospital Infantil de México Federico Gómez, Ciudad de México, México; ${ }^{2}$ Department of Pharmacology, \\ Korea University College of Medicine, Seúl, Corea del Sur; ${ }^{3}$ Unidad de Genómica de Poblaciones Aplicada a la Salud, Facultad de Química, \\ Universidad Nacional Autónoma de México, Ciudad de México, México; ${ }^{4}$ Instituto Nacional de Medicina Genómica, Ciudad de México, México; \\ ${ }^{5}$ División de Investigación, Facultad de Medicina, Universidad Nacional Autónoma de México, Ciudad de México, México
}

\section{Resumen}

En las últimas décadas, la prevalencia de la obesidad ha aumentado a escala mundial y ha provocado complicaciones metabólicas tales como diabetes de tipo 2, esteatosis, problemas cardiovasculares, entre otras. Su desarrollo puede estar influenciado por factores genéticos y ambientales, incluyendo la microbiota intestinal. En México, el 33.3\% de la población adulta padece de este problema. La obesidad involucra un incremento excesivo del tejido adiposo que produce su disfunción. Se ha demostrado que la remodelación del tejido adiposo, dada por angiogénesis, hipoxia e inflamación, otorga susceptibilidad a desarrollar obesidad y conlleva a modificaciones metabólicas. La cirugía bariátrica ha sido el procedimiento más utilizado y más exitoso para tratar la obesidad mórbida en cuanto a la pérdida de peso corporal a largo plazo y a la remisión de comorbilidades como la diabetes tipo 2. En este artículo se revisan los aspectos moleculares por medio de los cuales la cirugía bariátrica provoca cambios metabólicos que se reflejan en la pérdida de peso y las mejoras metabólicas. Por lo tanto, se resumen aspectos genéticos, de microbioma y moleculares (remodelación del tejido adiposo) que influyen en el desarrollo de la obesidad. También se explican los aspectos moleculares a través de los cuales se puede modular la pérdida de peso y la mejoría de comorbilidades metabólicas.

Palabras clave: Obesidad. Tejido adiposo. Cirugía bariátrica. Manga gástrica. Bypass gástrico.

\section{Obesity, adipose tissue, and bariatric surgery}

\begin{abstract}
Obesity prevalence has increased in the last decades worldwide leading to metabolic complications, such as type 2 diabetes, steatosis, cardiovascular disease, among others; its development is influenced by genetic factors and environmental factors, such as intestinal microbiome. In Mexico, 33.3\% of the adults present this disease. Obesity is defined as an excessive adipose tissue accumulation, provoking its dysfunction. Adipose tissue remodeling, which involves angiogenesis, hypoxia and inflammation, is implicated in the developing of obesity and metabolic modifications. Bariatric surgery is the most used and successful intervention to control morbid obesity, leading a maintained loss of weight and remission of some of its comorbidities as type 2 diabetes. Here, we review some of the molecular aspects of the metabolic changes provoked by bariatric surgery and its impact in weight loss and comorbidities remission. In summary, this article reviews the genetic aspects, microbiome and molecular facts (adipose tissue remodeling) that are involved in obesity development. In addition, some of the
\end{abstract}

\section{Correspondencia:}

*Ruth Gutiérrez-Aguilar

E-mail: ruthgutz@unam.mx
Disponible en internet: 17-02-2020 Bol Med Hosp Infant Mex. 2020;77(1):3-14 www.bmhim.com 1665-1146/@ 2019 Hospital Infantil de México Federico Gómez. Publicado por Permanyer. Este es un artículo open access bajo la licencia CC BY-NC-ND (http://creativecommons.org/licenses/by-nc-nd/4.0/). 
molecular aspects about bariatric surgery are described and the mechanisms that are regulated to control obesity and its comorbidities.

Key words: Obesity. Adipose tissue. Bariatric surgery. Sleeve gastrectomy. Gastric bypass.

\section{Introducción}

La Organización Mundial de la Salud (OMS) define la obesidad como una acumulación anormal o excesiva de grasa que puede ser perjudicial para la salud. En 2016, la OMS reportó que la prevalencia de la obesidad mundial en adultos era del $13 \%{ }^{1}$. En ese mismo año, la Encuesta Nacional de Salud y Nutrición de Medio Camino reportó que la prevalencia de obesidad en mexicanos adultos era del $33.3 \%{ }^{2}$.

La obesidad es la acumulación excesiva de grasa. El índice de masa corporal (IMC) es uno de los métodos para evaluar el estado nutricional de una persona y uno de los medidores antropométricos más utilizados. El IMC se obtiene al dividir el peso $(\mathrm{kg})$ entre el cuadrado de la estatura $\left(\mathrm{m}^{2}\right)$. La OMS estableció los puntos de corte (Tabla 1), los cuales indican que un IMC entre $18.5-24.9 \mathrm{~kg} / \mathrm{m}^{2}$ se considera como un índice normal, mientras que un IMC de $25-29.9 \mathrm{~kg} / \mathrm{m}^{2}$ es un indicador de sobrepeso. Si el IMC $\geq 30 \mathrm{~kg} / \mathrm{m}^{2}$, se clasifica como obesidad'1.

Por otra parte, se utilizan los criterios de la edad y el sexo con base en las curvas de crecimiento de la CDC (Center of Disease Control and Prevention) para la clasificación de sobrepeso y obesidad en niños ${ }^{3}$. Por lo tanto, el IMC se traslada a una tabla de percentiles. De esa forma, se clasifica como con sobrepeso a aquellos infantes que se encuentran entre el percentil 85 y 95. La obesidad en niños se define cuando se encuentran en un percentil mayor o igual a 95.

El sobrepeso y la obesidad confieren riesgo para desarrollar otras enfermedades no transmisibles, como la diabetes de tipo 2, esteatosis hepática, cardiopatías, trastornos del aparato locomotor, cáncer, entre otras. Otros problemas de salud también se han asociado con esta enfermedad, como problemas de la vesícula biliar y osteoartritis 4 . Una comorbilidad asociada con la obesidad es la diabetes tipo 2, que presenta una prevalencia del $9.4 \%$ en adultos mexicanos ${ }^{2}$.

La cirugía bariátrica ha sido el procedimiento más utilizado y más exitoso para tratar la obesidad mórbida en cuanto a la pérdida de peso corporal a largo plazo y a la mejoría de enfermedades asociadas, como la diabetes tipo 2, la hipertensión y la esteatosis hepática.

\section{Genómica de la obesidad}

El sobrepeso y la obesidad son provocados por un desequilibrio en la balanza energética, la cual se compone por los alimentos que se consumen (energía ingerida) y por la actividad física (energía gastada). Este desbalance puede ser provocado por factores ambientales como el exceso en la alimentación o la falta de actividad física. Sin embargo, también existen factores genéticos que confieren susceptibilidad a desarrollar esta enfermedad.

La obesidad se puede clasificar de acuerdo a su etiología en tres categorías: sindrómica, monogénica y poligénica o común ${ }^{5}$. La obesidad sindrómica presenta, además del fenotipo obesidad, problemas dismórficos, malformaciones de órganos y problemas cognitivos que se desarrollan después de la infancia. Algunos ejemplos de este tipo de obesidad son los síndromes de Prader-Willi, Bardet-Biedl, Alstrom, entre otros. La prevalencia de estos síndromes es baja y se han identificado los genes o regiones cromosómicas que se encuentran alterados ${ }^{6}$.

Se ha demostrado que solamente en el $5 \%$ de personas que padecen obesidad, la causa es la afección de un gen, lo que se conoce como obesidad monogénica. Este término hace referencia a un número de formas raras de obesidad severa como resultado de mutaciones en un gen o región cromosómica ${ }^{7}$. La característica principal de este tipo de obesidad es la hiperfagia severa, que causa un incremento de peso muy rápido en el primer año de vida. Varios de los genes implicados en este tipo de obesidad participan en el control de la balanza energética (ingesta de alimentos y gasto energético) en el hipotálamo. Se conocen ocho genes que conducen a formas monogénicas de obesidad severa de aparición precoz: leptina (LEP), receptor de la leptina (LEPR), proopiomelanocortina (POMC), preproconvertasa 1 (PCSK1), receptor de la melanocortina 4 (MC4R), single minded 1 (SIM1), factor neurotrófico derivado del cerebro (BDNF) y receptor de tropomiosina relacionado a la cinasa $\mathrm{B}(T R K B)^{8}$.

La obesidad «común» se debe a la implicación de varios genes, por lo que se denomina obesidad poligénica, además de ciertos factores ambientales que influyen en el desarrollo de la enfermedad ${ }^{7}$. Se ha 
Tabla 1. Clasificación del índice de masa corporal (IMC)

\begin{tabular}{|l|c|}
\hline Clasificación & IMC $\left(\mathrm{kg} / \mathrm{m}^{2}\right)$ \\
\hline Insuficiencia ponderal & $<18.5$ \\
\hline Intervalo normal & $18.5-24.9$ \\
\hline Sobrepeso & $25.0-29.9$ \\
\hline Obesidad & $\geq 30.0$ \\
\hline Obesidad clase I & $30.0-34.9$ \\
Obesidad clase II & $35.0-39.9$ \\
Obesidad clase III & $\geq 40.0$ \\
\hline
\end{tabular}

descrito la relación de estos genes con la obesidad mediante estudios de asociación: estudios de genes candidatos y estudios de asociación del genoma completo (GWAS, por sus siglas en inglés Genome wide association studies).

\section{Genes candidatos asociados con la obesidad}

En los estudios de asociación por genes candidatos, se seleccionan genes cuya función se conoce previamente, ya sea por estudios fisiológicos, moleculares o celulares. Al conocer la función del gen, se sabe su implicación en algún proceso fisiopatológico implicado en la enfermedad ${ }^{9}$. Por lo tanto, se infiere la posible asociación genética. Este abordaje tiene como limitante el conocimiento de los genes y sus funciones. Sin embargo, se han descubierto cerca de 430 genes y regiones cromosómicas implicadas en la obesidad por medio de este abordaje ${ }^{10}$. Algunos de los genes asociados con la obesidad descubiertos por el estudio de genes candidatos son LEP, LEPR, POMC, MC4R, PCSK1, adiponectina $(A D I P O Q)$, receptor-gama activado por proliferadores de peroxisomas (PPARG), ectonucleótido pirofosfatasa/fosfodiesterasa 1 (ENPP1) $)^{8,11-13}$.

\section{Asociación del genoma completo de obesidad}

La secuenciación del genoma humano permitió el descubrimiento de las variantes alélicas o polimorfismos que se encuentran presentes en el genoma. Estas variantes alélicas se refieren al cambio de una base nucleotídica del ácido desoxirribonucleico (ADN), diferentes entre el alelo del padre y de la madre, los cuales representan el $1 \%$ de la secuencia del genoma de cada individuo.

Los avances tecnológicos permitieron el desarrollo de nuevas metodologías que condujeron al cambio de la genética a la genómica. En la genética se estudiaban pocos genes asociados con una enfermedad, cuya función era conocida. Sin embargo, en la genómica se analizan millones de variantes en miles de genes de función y su asociación con alguna enfermedad desconocida.

La genómica se logró, principalmente, con el desarrollo de estudios de asociación del genoma completo. Estos estudios se realizan por medio de microarreglos, con los que se analizan millones de variantes alélicas presentes a lo largo del genoma. Todas estas variantes pueden analizarse al mismo tiempo, lo cual reduce el tiempo y costo de los estudios. Para ello, se utilizan cohortes muy grandes, comparando sujetos no relacionados, donde algunos individuos presentan la enfermedad (casos) y otros no la presentan (controles).

En 2009, se publicaron los primeros tres estudios de asociación del genoma completo de la obesidad. Estos estudios de asociación del genoma completo identificaron 16 loci asociados con la obesidad: FTO, MC4R, TMEM18, GNPDA2, SH2B1, MTCH2, KCTD15, NEGR1, ETV5, BDNF, SEC16B, FAIM2, NPC1, MAF, PTER y $P R L^{14-16}$. Actualmente, se conocen 119 loci asociados con el IMC y rasgos relacionados con la obesidad ${ }^{17}$.

La genómica permitió la apertura de un gran campo de investigación, con el fin de entender las variantes genéticas que confieren susceptibilidad para desarrollar la obesidad. Sin embargo, el reto continúa: comprender la forma en que esas variantes regulan diversos genes, describir la función de dichos genes y su implicación en el metabolismo. El presente grupo de trabajo lleva a cabo esta investigación ${ }^{18,19}$. Los estudios genómicos y a nivel molecular permitirán entender mejor la etiología de la obesidad.

\section{Microbioma e implicación en la obesidad}

Dentro de los factores biológicos que podrían asociarse con los cambios en adiposidad y, por ende, con un sobrepeso u obesidad, se encuentra la microbiota intestinal. La evidencia científica la sugiere como un factor biológico clave en la interfase entre la genética humana y la nutrición. La microbiota intestinal, la cual se compone de trillones de bacterias, influye de forma importante sobre la fisiología del hospedero y vive en simbiosis con el mismo. De hecho, le confiere capacidades metabólicas complementarias, como la fermentación de hidratos de carbono, metabolismo de aminoácidos, integridad del intestino, regulación de la respuesta inmunitaria, almacenamiento de lípidos en el tejido adiposo ${ }^{20}$. La microbiota intestinal está 
influenciada por diversos factores, como la genética del hospedero, la dieta, la edad, el ambiente, los antibióticos, el uso de pre- o probióticos. Estos factores pueden ocasionar un desbalance en su composición y función ${ }^{21}$. Las bacterias que se encuentran en el colon son capaces de fermentar fibras dietéticas y convertirlas en ácidos grasos de cadena corta (acetato, propionato y butirato), los cuales contribuyen a la síntesis de novo de glucosa y de lípidos ${ }^{22}$. Esto permite que las bacterias extraigan energía a partir de hidratos de carbono no digeribles y contribuyan a un balance energético positivo para el hospedero.

En los últimos años, se ha demostrado que la obesidad y sus complicaciones metabólicas se relacionan con la composición de la microbiota intestinal ${ }^{23}$. Se han observado modificaciones en la composición de la microbiota intestinal en la obesidad, principalmente en dos phyla: un aumento en Firmicutes y una disminución en Bacteroidetes. Además, se reportó una menor diversidad de bacterias ${ }^{24}$, considerándose una disbiosis en presencia de esta enfermedad. Por otra parte, los Firmicutes generan cambios epigenéticos (regulación de la estructura tridimensional del ADN y de la expresión de los genes), los cuales están asociados con un incremento del riesgo de enfermedades cardiovasculares, y alteran el metabolismo de los lípidos, y causan obesidad, inflamación y estrés oxidativo ${ }^{25}$. También se demostró que la composición de la microbiota intestinal predice la diabetes de tipo 2 mejor que el IMC ${ }^{26}$. En relación con las complicaciones derivadas de la obesidad, se han identificado cambios en los géneros Bifidobacterium, Akkarmansia, Faecalibacterium y Roseburia en sujetos con diagnóstico de diabetes y de hígado graso no alcohólico ${ }^{23,27}$. Asimismo, en sujetos obesos con síndrome metabólico se encontró que la disminución en Firmicutes está asociada con mayores concentraciones circulantes de ácidos biliares, lo cual produce una disminución en la sensibilidad a la insulina ${ }^{28}$. Por lo tanto, los cambios a nivel de composición taxonómica de la microbiota tienen influencia sobre el hospedero, modificando el perfil metabolómico ${ }^{29}$. Sin embargo, se requiere mayor investigación para comprender la microbiota intestinal y su interacción con el metabolismo humano, con el fin de proponer estrategias de alimentación y otras terapias para su modulación.

\section{Tejido adiposo como órgano endocrino}

El tejido adiposo se encuentra distribuido superficialmente de forma subcutánea y, más profundamente, de forma visceral. Contrariamente a lo que antes se pensaba, es un órgano sumamente dinámico y metabólicamente muy activo. Su función es almacenar lípidos en forma de triacilgliceroles para contar con una reserva de energía cuando esta no está disponible, como en el ayuno. Además, ahora se sabe que el tejido adiposo es un órgano endocrino, ya que expresa y libera gran cantidad de hormonas que tienen su efecto en el metabolismo energético global. El tejido adiposo está conformado por diversos tipos de células, como adipocitos, preadipocitos, fibroblastos, células del estroma vascular y macrófagos. Las hormonas que secreta el adipocito (célula funcional del tejido adiposo) ejercen su acción en el mismo adipocito, en células cercanas o en células de órganos distantes. Al ser liberadas por el adipocito, dichas hormonas se denominan adipocinas. Las adipocinas actúan para orquestar la homeostasis energética, entre otras funciones asociadas con dimorfismo sexual, inmunidad, reproducción, adipogénesis, angiogénesis, reestructuración de la matriz extracelular y mantenimiento de la temperatura corporal ${ }^{30}$.

La primera adipocina que se caracterizó fue la leptina. Esta hormona es principalmente secretada por los adipocitos del tejido adiposo blanco en condiciones de abundancia energética o durante el posprandio (periodo de dos horas después del consumo de alimento). La leptina regula la balanza energética y causa saciedad a nivel central al unirse a sus receptores en el núcleo arcuato del hipotálamo para promover la expresión de factores asociados con la sensación de saciedad (anorexigénicos) y detener la expresión de factores causantes del consumo de alimento (orexigénicos) ${ }^{31}$.

\section{Resistencia a la leptina}

Cuando la leptina se caracterizó, se pensó que podría funcionar como tratamiento contra la obesidad por causar saciedad. Sin embargo, se demostró que los animales e individuos obesos presentan altas concentraciones de leptina en suero. Esto significa que, a pesar de encontrarse en exceso en el suero de pacientes obesos, no puede ejercer su acción. Por lo tanto, en condiciones de obesidad, existe resistencia a la leptina, la cual se debe a una o varias irregularidades en la vía de la señalización de esta adipocina. Las vías de señalización se refieren a la respuesta fisiológica que promueve la unión de una señal a su receptor. En este caso, la leptina es la señal que, unida a su receptor, promueve la saciedad.

Las consecuencias de la falta de acción de la leptina o de la resistencia a la hormona en condiciones de obesidad son la hiperfagia, la lipotoxicidad y la resistencia 
a la insulina ${ }^{32}$. Los mecanismos moleculares por los cuales se produce la resistencia a la leptina se asocian con la inactividad del receptor de leptina, de la cinasa Jak o del factor STAT-3 (Signal Transducer and Activator of Transcription-3, entre otros intermediarios ${ }^{33}$.

En los últimos años, se ha investigado si el transporte de la leptina desde el tejido adiposo al cerebro (a través de la barrera hematoencefálica) se encuentra alterado en condiciones de resistencia a la leptina. Los hallazgos han mostrado que el transporte de la leptina no está alterado, ya que se obtuvieron las mismas concentraciones de leptina en el plexo coroidal (PC) y el centro mediobasal de ratones delgados y obesos (con resistencia a la leptina). Sin embargo, se halló una mayor expresión del receptor de leptina en el PC de ratones obesos con restricción energética $u$ otros tratamientos para disminuir el consumo de energía ${ }^{34}$. En congruencia con estos resultados, se ha demostrado un incremento del receptor de leptina en el tejido adiposo de humanos que se someten a pérdida de peso masiva después de la cirugía bariátrica con bypass gástrico en $Y$ de Roux ${ }^{35}$. Estos datos sugieren que la actividad del receptor y las moléculas intermediarias de la vía de señalización de la leptina integran el estado energético con las respuestas fisiológicas de hambre y saciedad.

Además de la leptina, la adiponectina es otra adipocina, que posee un peso molecular de $30 \mathrm{kDa}$ y forma multímeros (hexámeros y dodecámeros) ${ }^{36}$. Estas asociaciones de alto peso molecular se relacionan con su mayor actividad. Sus funciones engloban la oxidación de lípidos en músculo e hígado, la disminución de la expresión de moléculas de adhesión y citocinas proinflamatorias y la promoción de sensibilidad a la insulina. En humanos obesos, la hipoadiponectinemia se relaciona con obesidad, diabetes y síndrome metabólico ${ }^{37}$.

En resumen, el tejido adiposo contribuye de forma importante al metabolismo global a través de la síntesis de adipocinas, como la leptina y la adiponectina, las cuales, a su vez, regulan el consumo de alimento y utilización de energía.

\section{Remodelación del tejido adiposo y su implicación en homeostasis energética}

La obesidad involucra un incremento excesivo del tejido adiposo durante el cual se libera una mayor concentración de ácidos grasos libres y se desregula la síntesis de hormonas y péptidos de este tejido, lo que provoca una disfunción del mismo. El exceso de ácidos grasos causa que los adipocitos se vuelvan hipertróficos ${ }^{38}$, lo cual conlleva a que el tejido adiposo presente hipoxia, vascularización inadecuada, estrés e inflamación, lo que produce una reducción de su funcionalidad. Esta disfuncionalidad del tejido adiposo contribuye a la alteración de la síntesis de adipocinas, a un estado proinflamatorio, aterogénico y diabetogénico que ocasiona desregulación metabólica ${ }^{39}$.

En los últimos años, uno de los temas de interés ha sido los cambios del tejido adiposo durante la obesidad. Anteriormente, se demostró que la remodelación del tejido adiposo dada por la angiogénesis, hipoxia e inflamación otorga susceptibilidad a desarrollar la obesidad ${ }^{40}$. Se ha propuesto que la remodelación del tejido adiposo promueve modificaciones metabólicas. A este respecto, se observó que al reducir la vascularización (angiogénesis) en el tejido adiposo, utilizando un péptido proapoptótico que reduce el endotelio y la vascularización del tejido adiposo, disminuye el peso corporal, el porcentaje de masa grasa y el consumo de alimentos ${ }^{41}$. Además, la tolerancia a la glucosa mejora y las concentraciones de insulina y de triglicéridos disminuyen, con lo que se demuestra que la vascularización del tejido adiposo influye sobre la homeostasis de la glucosa y el metabolismo de lípidos ${ }^{42}$. También se ha estudiado que la hipoxia, la inflamación y la angiogénesis son otros factores que influyen para la remodelación del tejido adiposo y que otorgan susceptibilidad a desarrollar la obesidad ${ }^{40}$. Conjuntamente, se demostró que la remodelación del tejido adiposo es diferente dependiendo de los depósitos de tejido adiposo (visceral o subcutáneo) debido a la capacidad angiogénica, la inflamación, la hipoxia y la angiogénesis ${ }^{43}$.

Por lo tanto, una comprensión más profunda de la remodelación del tejido adiposo y su reestructuración durante la obesidad ayudará a entender mejor los mecanismos por los cuales se establece esta enfermedad.

\section{Cirugía bariátrica}

En el humano, existen diferentes tratamientos para el control de la obesidad. Los programas de intervención de dieta y actividad física resultan en una pérdida del peso corporal del $5-10 \%{ }^{44}$. Por otra parte, existen pocos medicamentos aprobados y tienen una eficacia limitada para el tratamiento de la obesidad ${ }^{45}$. Sin embargo, la cirugía bariátrica ha sido el procedimiento más utilizado y exitoso en cuanto a la pérdida de peso corporal y al mantenimiento de dicha pérdida a largo plazo. Además, la cirugía bariátrica ayuda a la resolución de comorbilidades como la diabetes tipo 2, la hipertensión y la esteatosis hepática ${ }^{46}$. 


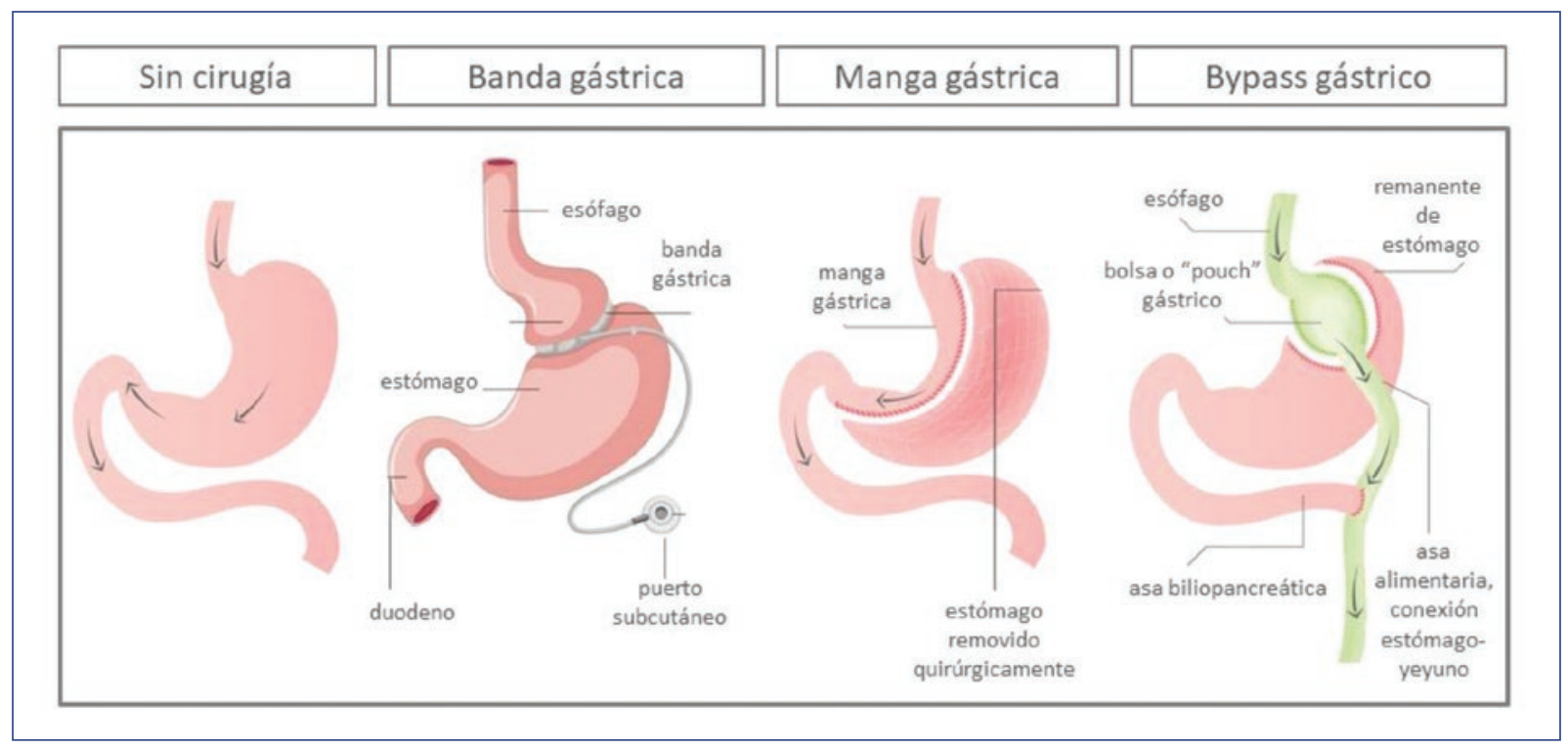

Figura 1. Esquematización de los procedimientos bariátricos. La banda gástrica promueve menor volumen gástrico y menor consumo de alimento sin cambios en las estructuras del estómago o el intestino. La manga gástrica genera la reducción del $80 \%$ del volumen gástrico. La derivación gastroyeyunal en Y de Roux o el bypass gástrico reducen el tamaño del estómago, dejando solamente una bolsa o pouch gástrico que se conecta al yeyuno y excluye el paso del alimento por el duodeno. Las imágenes anteriores fueron obtenidas y adaptadas a partir de https://bancosdeimagenes.com/agencias/ shutterstock/. Todo su contenido es libre de derechos, bajo licencia Libre de Regalías.

El tratamiento quirúrgico para la obesidad se ha considerado válido en adolescentes y adultos con IMC $\geq 40 \mathrm{~kg} / \mathrm{m}^{2}$ o con IMC = $35 \mathrm{~kg} / \mathrm{m}^{2}$ con comorbilidades asociadas a la obesidad ${ }^{47}$. Con respecto a la población pediátrica, la Norma Oficial Mexicana (NOM-008-SSA3-2017) establece que la cirugía bariátrica solamente está indicada en pacientes de 16 a 18 años con obesidad, que no hayan tenido una respuesta favorable al tratamiento integral y que hayan concluido su desarrollo físico y sexual ${ }^{47}$. Por estas razones, el equipo multidisciplinario debe atender los criterios para que ningún individuo menor de 16 años de edad o con un desarrollo incompleto se someta a un procedimiento quirúrgico para el tratamiento de la obesidad.

Se considera que una cirugía bariátrica exitosa es aquella donde existe una pérdida de al menos el $50 \%$ del exceso de peso corporal, sin recuperación de peso durante el año posquirúrgico ${ }^{48}$.

\section{Tipos de cirugía bariátrica}

Las técnicas más utilizadas para la cirugía bariátrica son la banda gástrica, la manga gástrica y la derivación gastroyeyunal en $Y$ de Roux o bypass gástrico ${ }^{49}$.
La banda gástrica consiste en colocar una banda inflable en la parte superior del estómago, lo que provoca que disminuya la entrada de la comida (Fig. 1). Esta banda puede ser reajustable y el procedimiento es reversible. Además, no existen cambios físicos en el estómago ni en los intestinos. La cirugía es considerada de bajo riesgo y la estancia en el hospital es corta. Algunas de las desventajas son la menor pérdida de peso en comparación con la manga gástrica o el bypass gástrico, la necesidad de acudir al médico a que les reajusten la banda, y una posible segunda cirugía para cambiar la banda o quitarla. Anteriormente, se consideraba una cirugía «restrictiva», ya que reduce la porción de comida ingerida; sin embargo, más adelante se aclara por qué esta cirugía ya no se debe de considerar «restrictiva».

La manga gástrica consiste en remover el $80 \%$ del estómago, dejando intactos el píloro y los intestinos. Esta cirugía también se consideraba como «restrictiva", ya que disminuye la capacidad gástrica. Sin embargo, el estómago es un músculo que puede volver a crecer. Las ventajas de la manga gástrica son que conlleva una pérdida de peso mayor a la banda gástrica y la estancia en el hospital es corta. En contraste, algunas desventajas de esta cirugía son que no es reversible, puede provocar reflujo ácido, un mayor 
riesgo de problemas relacionados con la cirugía comparado con la banda gástrica, entre otras (Fig. 1).

La cirugía de la derivación gastroyeyunal en $Y$ de Roux o bypass gástrico consiste en unir la parte superior del estómago con el yeyuno. De esta forma, se evita el duodeno, por lo que la comida cambiará su ruta, ya que entrará por la boca, seguirá por el esófago pasando por la pequeña bolsa del estómago, o "pouch", y después se irá hacia el yeyuno, que ha sido unido, sin pasar por el duodeno (Fig. 1). A esta cirugía se la conocía como mixta, ya que tiene un aspecto «restrictivo", pero también «malabsorbativo». En realidad, tanto la manga gástrica como el bypass gástrico, más que ser cirugías con efectos «restrictivos», muestran implicaciones metabólicas importantes, por lo que el término «restrictivo» no debería utilizarse para estas cirugías.

Después de diferentes tipos de cirugía bariátrica, la pérdida de peso al cabo de un año poscirugía se ha reportado de alrededor del $20 \%$ para la banda gástrica y del $32 \%$ para el bypass gástrico. A los 10 años poscirugía, el seguimiento de estos pacientes demostró que la pérdida había disminuido al 14\% para la banda gástrica y al $25 \%$ para el bypass gástrico ${ }^{50,51}$.

Un metaanálisis más reciente recopiló la información de 87 estudios que midieron la pérdida del exceso de peso (PEP) después de los diferentes procedimientos bariátricos. EI PEP se refiere al peso excedente representado a partir del IMC de $25 \mathrm{~kg} / \mathrm{m}^{2}$. Los resultados mostraron que el porcentaje de PEP fue del $47.94 \%$ para la banda gástrica y del $53.25 \%$ para la manga gástrica después de 5 años de la cirugía bariátrica ${ }^{52}$.

Recientemente, la Federación Internacional de Cirugía Bariátrica (IFSO, International Federation for the Surgery of Obesity) conjuntó los datos de cirugías llevadas a cabo en el periodo 2014-2018. Este estudio reunió información de 51 países, entre ellos México, y reportó que el $73.7 \%$ de los pacientes bariátricos son mujeres. La mediana del IMC precirugía fue de $41.7 \mathrm{~kg} / \mathrm{m}^{2}$ y la gran mayoría de estos pacientes presentaban comorbilidades ${ }^{53}$.

Globalmente, se realizaron 394,431 cirugías bariátricas entre 2014-2018: el 5\% para banda gástrica, el $46 \%$ para manga gástrica y el $38.2 \%$ para bypass gástrico ${ }^{53}$. En México, los informes del Colegio Mexicano de Cirugía para la Obesidad y Enfermedades Metabólicas, A.C. (CMCOEM) muestran que se realizaron un total de 7,784 cirugías bariátricas en 2016, de las cuales 121 (1.55\%) fueron procedimientos de banda gástrica, 4,235 (54.4\%) de manga gástrica y $1,752(22.50 \%)$ de bypass gástrico ${ }^{54}$. El resto de cirugías $(1,676)$ fueron de otro tipo, como bypass gástrico con anastomosis, SADI-S (por sus siglas en inglés, single anastomosis duodeno-ileal bypass with sleeve gastrectomy), plicatura gástrica, procedimientos endoluminales o cambio duodenal.

Además de la pérdida de peso, se ha documentado que estas técnicas quirúrgicas representan beneficios para el paciente, como mejoras en la sensibilidad a la insulina o remisión de la diabetes y esteatosis hepáti$\mathrm{ca}^{55}$. Se ha reportado que el $66.1 \%$ de los pacientes ya no recurrieron a los medicamentos hipoglucemiantes ${ }^{53}$. Por esto, también se le ha llamado cirugía metabólica, ya que ayuda a controlar la diabetes tipo 2. La mejora en la homeostasis de la glucosa podría deberse a la pérdida de peso 0 al incremento de las incretinas o modificación de sales biliares y de la microbiota ${ }^{56}$, temas que se abordarán más adelante.

Después de la cirugía bariátrica, los adipocitos disminuyen de tamaño $0^{57,58}$ y alcanzan casi el tamaño de sujetos delgados ${ }^{57}$, aunque el número de adipocitos no cambia ${ }^{59}$. Asimismo, se ha observado una mayor sensibilidad a la lipólisis controlada por catecolaminas en el tejido adiposo: la modificación de la secreción de adipocinas aumenta la sensibilidad a la insulina y disminuye la inflamación en el tejido adiposo, lo que beneficia el metabolismo ${ }^{60}$.

Sin embargo, además de las modificaciones del tejido adiposo, varios de los mecanismos por los cuales la cirugía bariátrica ejerce los beneficios de pérdida de peso y sobre las comorbilidades aún no han sido elucidados, por lo cual son motivo de estudio.

\section{Aspectos moleculares de la cirugía bariátrica}

En la última década, se han logrado grandes avances para la comprensión de los mecanismos moleculares por los cuales la cirugía bariátrica provoca la reducción de peso y mejorías de algunas comorbilidades de la obesidad.

Anteriormente, la cirugía bariátrica se definía como una intervención «restrictiva» o «malabsorbativa». Sin embargo, se ha demostrado que estos adjetivos no son adecuados, ya que estas cirugías no son solamente procesos físicos (restrictivo) o químicos (malabsorbativo), sino que provocan cambios metabólicos que generan los beneficios posquirúrgicos observados. Es decir, se pueden considerar como cirugías metabólicas. Para entender mejor los procesos moleculares involucrados en la cirugía bariátrica, se han utilizado modelos animales que han sido revisados ampliamente ${ }^{61,62}$. A continuación, se describen los procesos moleculares de las 
cirugías de manga gástrica y bypass gástrico, debido a que son las cirugías más utilizadas.

La manga gástrica y el bypass gástrico se definían como cirugías restrictivas, ya que en la manga gástrica se extraía el $80 \%$ del estómago y en el bypass gástrico solamente quedaba una pequeña bolsa del estómago unida al yeyuno. Estas intervenciones serían restrictivas porque se reduce el tamaño del estómago, se genera un pequeño espacio para almacenar la comida y se impide que el paciente se sobrealimente. Sin embargo, en modelos murinos, si los animales se someten a una restricción de alimentos y después regresan a una dieta ad libitum, los animales bariátricos son capaces de aumentar su consumo, lo que indica que la cirugía no es restrictiva ${ }^{63}$. Por otra parte, en otras condiciones fisiológicas, como la lactancia, se comprobó que los animales bariátricos pueden aumentar su consumo para poder adquirir el aporte energético necesario para lactar a sus crías ${ }^{64}$. Por lo tanto, sí son capaces de ingerir un mayor volumen de alimento.

La cirugía $Y$ de Roux o bypass gástrico, además de considerase «restrictiva», también ha sido descrita como «malabsorbativa», ya que la ruta por la que los alimentos transitan normalmente es modificada, debido a que no pasan ni por el estómago ni por el duodeno. Esto implica que ciertas sustancias no se puedan absorber (p.ej., grasas, vitaminas liposolubles), por lo que la energía ingerida disminuye ${ }^{65}$, y que existan deficiencias de algunos nutrimentos inorgánicos y orgánicos, como hierro y vitamina $D^{66}$. Sin embargo, se ha observado que la reducción de la energía ingerida no podría explicar la pérdida de peso después de la cirugía ${ }^{65}$. Por otra parte, a pesar que la manga gástrica solamente reduce el tamaño del estómago, pero no cambia la ruta de los alimentos, en modelo animal se comprobó que no existe malabsorción después de la cirugía bariátrica ${ }^{63}$. Por lo tanto, se ha establecido que tanto la manga gástrica como el bypass gástrico no son cirugías malabsorbativas ${ }^{62}$.

En modelos murinos, se ha observado que se reduce el consumo de alimento durante las primeras semanas después de la cirugía bariátrica, lo que provoca una disminución del peso. Sin embargo, al cabo de un tiempo, los animales vuelven a consumir la misma cantidad de alimento que consumían antes de la cirugía ${ }^{63}$. Por lo tanto, después de estas cirugías, los animales son capaces de mantener el nuevo peso corporal, a pesar de consumir nuevamente la misma cantidad de alimento. Se han realizado otros experimentos donde, por medio de restricción alimentaria, los animales pierden peso aún más de lo que ya habían perdido por la cirugía. Al quitar la restricción, estos animales son capaces de aumentar su ingesta calórica con el fin de alcanzar el peso predispuesto después de la cirugía, por lo que mantienen el nuevo peso corporal ${ }^{63}$.

Después de la cirugía bariátrica, se ha reportado que los pacientes presentan menor apetito, lo que causa que la balanza energética sea negativa, y así se produzca la pérdida de peso ${ }^{67,68}$.

La cuantificación de la ingesta de alimento y la composición de los alimentos ingeridos es difícil de calcular, ya que es posible que los pacientes no reporten todo lo que consumen ni las porciones ingeridas. Además, los seres humanos pueden ser influenciados por las sugerencias que reciben para la nueva dieta y el cambio de rutinas. Sin embargo, en modelos murinos, esta cuantificación se puede lograr, ya que los animales se alimentan en respuesta a las señales biológicas. Por ello, el empleo de modelos animales para el estudio de las preferencias posquirúrgicas de alimentos ha aportado valiosa información. Para estudiar la preferencia posquirúrgica de alimentos, se cuantificó la cantidad y la frecuencia de alimento que consumían los ratones después de la cirugía. Durante las primeras semanas postoperatorias, se observó que los animales consumieron menos alimento y se mantuvo el nuevo peso. Sin embargo, en semanas posteriores, se observó que estos animales consumían lo mismo que los controles, pero el patrón de comidas era diferente, ya que ingerían una menor cantidad de alimento, pero más frecuentemente ${ }^{63}$. También se ha reportado que la preferencia por los hidratos de carbono, lípidos y proteínas cambia después de la cirugía. Al tener un estómago más reducido, se esperaba que los ratones consumieran alimento con mayor densidad (mayor cantidad de grasa) para obtener la misma carga energética. Sin embargo, se observó que existe una preferencia por un mayor consumo de carbohidratos en comparación con lípidos y proteínas ${ }^{69}$. Esto indica que existen cambios fisiológicos que obligan a los animales a ajustar el patrón de consumo y la preferencia de alimentos y nutrimentos. En humanos, también se ha reportado que existe una reducción en la ingesta de alimentos hiperenergéticos ${ }^{70}$.

Por otra parte, se ha observado que la microbiota cambia después de la cirugía bariátrica: se reduce la relación de Firmicutes/Bacteriodetes tanto en humanos como en modelos animales ${ }^{71,72}$. Se ha sugerido que la microbiota está implicada en la pérdida de peso después de la cirugía bariátrica. Esto se ha comprobado mediante el trasplante de heces de animales con bypass gástrico a animales libres de gérmenes, lo que produjo una reducción de peso $^{73}$. Sin embargo, es 
necesario realizar más estudios para entender el mecanismo mediante el cual la microbiota ejerce el efecto beneficioso después de la cirugía bariátrica.

Se sabe que la microbiota está implicada en la regulación de la composición de los ácidos biliares. Las sales biliares son producidas en el hígado, se almacenan en la vesícula biliar $\mathrm{y}$, posteriormente, son secretadas al duodeno, donde se mezclan con el bolo alimenticio para permitir la absorción de lípidos (triglicéridos, colesterol) y vitaminas liposolubles. Después de una cirugía de bypass gástrico, se ha observado que la cantidad de sales biliares incrementa y su composición cambia en humanos, ratas y cerdos ${ }^{74}$. En ratones, también se ha reportado que, después de la colocación de una manga gástrica, incrementan las sales biliares ${ }^{75}$.

Además de participar en el proceso de absorción de grasas, los ácidos biliares activan al receptor $X$ farnesoide ( $F X R$, de sus siglas en inglés Farnesoid $X$ receptor) y al receptor acoplado a proteína G (TGR5) ${ }^{76,77}$. La activación de FXR incrementa los niveles del factor de crecimiento de fibroblastos (FGF19), encargado de la conversión del colesterol a ácidos biliares. El FGF19 tiene efectos metabólicos, tales como la reducción de la ingesta de alimento, reducción de peso y la regulación de la homeostasis de la glucosa ${ }^{78}$. En un estudio, se colocó la manga gástrica en ratones deficientes de FXR y se observó que existen cambios en la microbiota después de la cirugía, tanto en estos ratones como en los ratones silvestres. De forma interesante, los ratones deficientes de FXR disminuyeron el efecto de la manga gástrica en cuanto a la pérdida de peso y mejora de la tolerancia a la glucosa ${ }^{72}$. Estos resultados resaltan la vía de señalización de FXR como un factor responsable para la pérdida de peso después de la manga gástrica, que impacta en los niveles de ácidos biliares, la microbiota y el control de la homeostasis de la glucosa. Por otra parte, se ha observado que los ácidos biliares activan a GPBAR1 (G-Protein-coupled Bile Acid Receptor 1 or TGR5), el cual a su vez activa la secreción de GLP1 (péptido similar al glucagón) ${ }^{77}$, posible mecanismo por el cual se revierte la diabetes tipo 2.

Después de la cirugía de manga gástrica o bypass gástrico, se ha observado un aumento en las concentraciones de GLP179-81. El GLP1 es una incretina que ayuda a la secreción de insulina. Anteriormente, se postulaba que el GLP1 podría ser una de las razones por las cuales se observaba una remisión de la diabetes tipo 2 después de la cirugía bariátrica ${ }^{82}$. Sin embargo, diversos estudios demostraron que los beneficios observados después de estas cirugías no son mecanismos tan simples. Después de la cirugía bariátrica, los niveles de glucosa en ayuno se reducen y se mejora la tolerancia a la glucosa, lo que contribuye a la remisión de la diabetes tipo $2^{55,80}$. Se podría pensar que, debido a la pérdida de peso provocada por la cirugía bariátrica, los niveles de glucosa en sangre disminuyen también. Sin embargo, se ha comprobado que la remisión de la diabetes tipo 2 es independiente de la pérdida de peso $^{79}$, lo cual indica que existen cambios metabólicos que ayudan al control de la homeostasis de la glucosa. Por consiguiente, los niveles elevados de GLP1 posquirúrgicos se consideraban como un buen indicador para la mejora de la homeostasis de la glucosa. Esta idea se sustentó al administrar antagonistas del receptor de GLP1 (GLP1R), así se bloqueaba la acción de GLP1 sobre su receptor y, como consecuencia, se impedía la secreción de la insulina, lo que provocó que no se mejorara la tolerancia a la glucosa en los pacientes bariátricos ${ }^{83,84}$. Sin embargo, al realizar las cirugías bariátricas en animales deficientes de los receptores de GLP1 (KO-GLP1R), se observó que no existe diferencia entre los animales KO (knock-out) y los silvestres, lo que descarta la implicación del GLP1R en los beneficios provocados por ambas cirugías ${ }^{85,86}$.

La remisión de la diabetes tipo 2 en humanos y la mejoría de la intolerancia a la glucosa en modelos animales después de la cirugía bariátrica son el motivo por el cual se conoce a estas cirugías como cirugías metabólicas. Esto se debe a los cambios fisiológicos que suceden en el metabolismo. Como se mencionó anteriormente, la mejoría en la intolerancia a la glucosa es independiente de la pérdida de peso ${ }^{79}$. Se ha reportado un incremento de insulina después de la cirugía bariátrica $^{87,88}$. Además, se ha observado que el tránsito del bolo de glucosa es más rápido después de la cirugía bariátrica, lo que provoca que se estimule la secreción de GLP1 y ejerza su efecto sobre las células beta del páncreas para la estimulación de la insulina ${ }^{89}$. Por otra parte, se ha demostrado que se incrementa la sensibilidad a la insulina hepática, independientemente de la pérdida de peso ${ }^{79,90}$. Sin embargo, en una fase más tardía, se incrementa la sensibilidad de la insulina en músculo, debido a la pérdida de peso ${ }^{91}$. Por lo tanto, la remisión de la diabetes se debe tanto a un incremento en la secreción de la insulina como a una mayor sensibilidad de la insulina a nivel hepático ${ }^{92}$.

Otros genes candidatos para explicar los mecanismos moleculares de la cirugía bariátrica fueron el MC4R y la grelina. El MC4R es un gen del sistema nervioso central que regula la balanza energética ${ }^{93,94}$. Las mutaciones en el MC4R han sido descritas como 
la causa más frecuente de la obesidad monogéni$\mathrm{ca}^{95,96}$. Estudios realizados en ratas deficientes de $M C 4 R$ indicaron que los beneficios observados después de la cirugía bariátrica no son regulados por $M C 4 R^{97}$. Por otra parte, se estudió la influencia de la grelina en la cirugía bariátrica. La grelina es una hormona orexigénica (estimula la ingesta de alimentos) secretada a partir de las células del estómago ${ }^{98}$. Debido a que el $80 \%$ del estómago es removido durante la manga gástrica, existiría una disminución de la secreción de la grelina que provocaría una disminución de la ingesta de alimentos y, por ende, pérdida de peso. Se realizó la cirugía de manga gástrica en ratones deficientes de grelina, y se observó que los efectos de la cirugía bariátrica eran independientes de la grelina ${ }^{99}$. Por lo tanto, actualmente, el único gen que ha podido explicar su implicación en los cambios metabólicos después de la cirugía bariátrica ha sido $\mathrm{FXR}^{72}$.

La obesidad afecta a gran parte de la población. Existen factores genéticos y ambientales que influyen en el desarrollo de esta enfermedad. Gracias a los avances tecnológicos de los últimos años, se han descubierto nuevos genes asociados con la obesidad. Sin embargo, se necesita describir la función de estos genes y sus variantes, para comprender su implicación en esta enfermedad.

Durante el establecimiento del sobrepeso y la obesidad, el tejido adiposo se vuelve disfuncional y provoca una desregulación metabólica. Por esto, es necesario estudiar más a detalle el tejido adiposo, su distribución y remodelación, para entender sus implicaciones dentro del metabolismo. La cirugía bariátrica ha sido la intervención más exitosa para el tratamiento de la obesidad y sus comorbilidades. Recientemente, se han descrito algunos de los mecanismos por los que estas cirugías modulan el metabolismo y ayudan a la pérdida de peso. Sin embargo, es necesario continuar investigando sobre los mecanismos moleculares que influyen en el mantenimiento del nuevo peso corporal.

Actualmente, el presente grupo de trabajo se encuentra estudiando la remodelación del tejido adiposo (angiogénesis, hipoxia e inflamación) después de la cirugía bariátrica, para contribuir al conocimiento de los mecanismos moleculares que esta cirugía modifica, provocando así las mejoras metabólicas.

\section{Responsabilidades éticas}

Protección de personas y animales. Los autores declaran que para esta investigación no se han realizado experimentos en seres humanos ni en animales.
Confidencialidad de los datos. Los autores declaran que en este artículo no aparecen datos de pacientes.

Derecho a la privacidad y consentimiento informado. Los autores declaran que en este artículo no aparecen datos de pacientes.

\section{Conflicto de intereses}

Los autores declaran no tener ningún conflicto de intereses.

\section{Financiamiento}

CONACYT-NRF 2016 proyecto No. 278791, PAPIIT-DGAPA-UNAM proyecto No. IA203919, HIM-2018028FF SSA1494, IBM Mexico, National Research Foundation of Korea (NRF-2016K2A9A1A06945430), Basic Science Research Program through the National Research Foundation of Korea (2017R1D1A1B03031216).

\section{Bibliografía}

1. World Health Organization; 2018. Disponible en: https://www.who.int/en/ news-room/fact-sheets/detail/obesity-and-overweight.

2. Shamah Levy T, Cuevas Nasu L, Rivera Dommarco J, Hernández Ávila M, coordinadores. Encuesta Nacional de Salud y Nutrición de Medio Camino 2016. Informe final de resultados. Instituto Nacional de Salud Pública, Secretaría de Salud; 2016.

3. Centers for Disease Control and Prevention. Division of Nutrition, Physical Activity, and Obesity, National Center for Chronic Disease Prevention and Health Promotion; 2019.

4. Sánchez-Castillo CP, Pichardo-Ontiveros E, López RP. Epidemiología de la obesidad. Gac Med Mex. 2004;140:S3-20.

5. Albuquerque D, Stice E, Rodríguez-López R, Manco L, Nóbrega C. Current review of genetics of human obesity: from molecular mechanisms to an evolutionary perspective. Mol Genet Genomics. 2015;290:1191-221.

6. Mason K, Page L, Balikcioglu PG. Screening for hormonal, monogenic, and syndromic disorders in obese infants and children. Pediatr Ann. 2014; 43:e218-24.

7. El-Sayed Moustafa JS, Froguel P. From obesity genetics to the future of personalized obesity therapy. Nat Rev Endocrinol. 2013;9:402-13.

8. Ramachandrappa S, Farooqi IS. Genetic approaches to understanding human obesity. J Clin Invest. 2011;121:2080-6.

9. Tabor HK, Risch NJ, Myers RM. Candidate-gene approaches for studying complex genetic traits: practical considerations. Nat Rev Genet. 2002;3:391-7.

10. Snyder EE, Walts B, Pérusse L, Chagnon YC, Weisnagel SJ, Rankinen T, et al. The human obesity gene map: the 2003 update. Obes Res. 2004;12:369-439.

11. Ghoussaini M, Meyre D, Lobbens S, Charpentier G, Clément K, Charles MA, et al. Implication of the Pro12Ala polymorphism of the PPAR-gamma 2 gene in type 2 diabetes and obesity in the French population. BMC Med Genet. 2005;6:11.

12. Bouatia-Naji N, Meyre D, Lobbens S, Seron K, Fumeron F, Balkau B, et al. ACDC/adiponectin polymorphisms are associated with severe childhood and adult obesity. Diabetes. 2006;55:545-50.

13. Meyre D, Bouatia-Naji N, Vatin V, Veslot J, Samson C, Tichet J, et al. ENPP1 K121Q polymorphism and obesity, hyperglycaemia and type 2 diabetes in the prospective DESIR Study. Diabetologia. 2007;50:2090-6.

14. Willer CJ, Speliotes EK, Loos RJF, Li S, Lindgren CM, Heid IM, et al. Six new loci associated with body mass index highlight a neuronal influence on body weight regulation. Nat Genet. 2009;41:25-34.

15. Thorleifsson G, Walters G.B., Gudbjartsson DF, Steinthorsdottir V, Sulem P, Helgadottir A, et al. Genome-wide association yields new sequence variants at seven loci that associate with measures of obesity. Nat Genet. 2009;41:18-24.

16. Meyre D, Delplanque J, Chèvre JC, Lecoeur C, Lobbens S, Gallina S, et al. Genome-wide association study for early-onset and morbid adult obesity identifies three new risk loci in European populations. Nat Genet. 2009;41:157-9. 
17. Locke AE, Kahali B, Berndt SI, Justice AE, Pers TH, Day FR, et al. Genetic studies of body mass index yield new insights for obesity biology. Nature. 2015;518:197-206.

18. Gutierrez-Aguilar R, Kim DH, Woods SC, Seeley RJ. Expression of new loci associated with obesity in diet-induced obese rats: from genetics to physiology. Obes (Silver Spring). 2012;20:306-12.

19. Gutierrez-Aguilar R, Kim DH, Casimir M, Dai XQ, Pfluger PT, Park J, et al. The role of the transcription factor ETV5 in insulin exocytosis. Diabetologia. 2014;57:383-91.

20. Andoh A. Physiological role of gut microbiota for maintaining human health. Digestion. 2016;93:176-81.

21. Cani $P$, Delzenne $N$. The role of the gut microbiota in energy metabolism and metabolic disease. Curr Pharm Des. 2009;15:1546-58.

22. Carvalho BM, Abdalla Saad MJ. Influence of gut microbiota on subclinical inflammation and insulin resistance. Mediators Inflamm. 2013:2013:1-13.

23. Karlsson F, Tremaroli V, Nielsen J, Bäckhed F. Assessing the human gut microbiota in metabolic diseases. Diabetes. 2013;62:3341-9.

24. Hildebrandt MA, Hoffmann C, Sherrill-Mix SA, Keilbaugh SA, Hamady M, Chen $Y Y$, et al. High-fat diet determines the composition of the murine gut microbiome independently of obesity. Gastroenterology. 2009;137:1716-24

25. Kumar H, Lund R, Laiho A, Lundelin K, Ley RE, Isolauri E, et al. Gut microbiota as an epigenetic regulator: pilot study based on whole-genome methylation analysis. MBio. 2014;5: pii: e02113-14.

26. Karlsson FH, Tremaroli V, Nookaew I, Bergström G, Behre CJ, Fagerberg B, et al. Gut metagenome in European women with normal, impaired and diabetic glucose control. Nature. 2013;498:99-103.

27. Cotillard A, Kennedy SP, Kong LC, Prifti E, Pons N, Le Chatelier E, et al. Dietary intervention impact on gut microbial gene richness. Nature. 2013:500:585-8.

28. Vrieze A, Out C, Fuentes S, Jonker L, Reuling I, Kootte RS, et al. Impact of oral vancomycin on gut microbiota, bile acid metabolism, and insulin sensitivity. J Hepatol. 2014;60:824-31.

29. Nicholson JK, Holmes E, Kinross J, Burcelin R, Gibson G, Jia W, et al Host-gut microbiota metabolic interactions. Science. 2012;336:1262-7.

30. Unamuno X, Gómez-Ambrosi J, Rodríguez A, Becerril S, Frühbeck G Catalán V. Adipokine dysregulation and adipose tissue inflammation in human obesity. Eur J Clin Invest. 2018;48:e12997.

31. Crujeiras AB, Carreira MC, Cabia B, Andrade S, Amil M, Casanueva FF. Leptin resistance in obesity: An epigenetic landscape. Life Sci. 2015 140:57-63.

32. Unger RH, Scherer PE. Gluttony, sloth and the metabolic syndrome: a roadmap to lipotoxicity. Trends Endocrinol Metab. 2010;21:345-52.

33. Foster-Schubert KE, Cummings DE. Emerging therapeutic strategies for obesity. Endocr Rev. 2006;27:779-93.

34. Harrison L, Schriever SC, Feuchtinger A, Kyriakou E, Baumann P, Pfuhlmann $\mathrm{K}$, et al. Fluorescent blood-brain barrier tracing shows intact leptin transport in obese mice. Int J Obes (Lond). 2018:43:1305-18.

35. Tamez M, Ramos-Barragan V, Mendoza-Lorenzo P, Arrieta-Joffe P, López-Martínez S, Rojano-Rodríguez ME, et al. Adipocyte size and leptin receptor expression in human subcutaneous adipose tissue after roux-en-y gastric bypass. Obes Surg. 2017;27:3330-2

36. Kadowaki T, Yamauchi T, Kubota N, Hara K, Ueki K, Tobe K. Adiponectin and adiponectin receptors in insulin resistance, diabetes, and the metabolic syndrome. J Clin Invest. 2006;116:1784-92.

37. Yatagai $T$, Nagasaka $S$, Taniguchi A, Fukushima M, Nakamura $T$, Kuroe $A$, et al. Hypoadiponectinemia is associated with visceral fat accumulation and insulin resistance in Japanese men with type 2 diabetes mellitus. Metabolism. 2003;52:1274-8

38. Spalding KL, Arner E, Westermark PO, Bernard S, Buchholz BA, Bergmann O, et al. Dynamics of fat cell turnover in humans. Nature. 2008;453:783-7.

39. Blüher M. Adipose tissue dysfunction contributes to obesity related metabolic diseases. Best Pract Res Clin Endocrinol Metab. 2013;27:163-77.

40. Kim DH, Gutierrez-Aguilar R, Kim HJ, Woods SC, Seeley RJ. Increased adipose tissue hypoxia and capacity for angiogenesis and inflammation in young diet-sensitive C57 mice compared with diet-resistant FVB mice. Int J Obes (Lond). 2013;37:853-60.

41. Kim DH, Woods SC, Seeley RJ. Peptide designed to elicit apoptosis in adipose tissue endothelium reduces food intake and body weight. Diabetes. 2010;59:907-15.

42. Kim DH, Sartor MA, Bain JR, Sandoval D, Stevens RD, Medvedovic M, et al. Rapid and weight-independent improvement of glucose tolerance induced by a peptide designed to elicit apoptosis in adipose tissue endothelium. Diabetes. 2012;61:2299-310

43. Song M-G, Lee HJ, Jin BY, Gutierrez-Aguilar R, Shin $\mathrm{KH}$, Choi SH, et al Depot-specific differences in angiogenic capacity of adipose tissue in differential susceptibility to diet-induced obesity. Mol Metab. 2016;5:1113-20.

44. Kraschnewski JL, Boan J, Esposito J, Sherwood NE, Lehman EB Kephart DK, et al. Long-term weight loss maintenance in the United States. Int J Obes (Lond). 2010;34:1644-54

45. Bray GA. Lifestyle and pharmacological approaches to weight loss: efficacy and safety. J Clin Endocrinol Metab. 2008:93:s81-8.

46. Schauer PR, Bhatt DL, Kirwan JP, Wolski K, Brethauer SA, Navaneethan SD, et al. Bariatric surgery versus intensive medical therapy for diabetes - 3-year outcomes. N Engl J Med. 2014;370:2002-13.
47. Norma Oficial Mexicana NOM-008-SSA3-2010, Para el tratamiento integral del sobrepeso y la obesidad. Diario Oficial de la Federación; 2010.

48. Brethauer SA, Kim J, el Chaar M, Papasavas P, Eisenberg D, Rogers A, et al. Standardized outcomes reporting in metabolic and bariatric surgery. Obes Surg Relat Dis. 2015;11:489-506.

49. Buchwald H. Laparoscopic Roux-en-Y Gastric Bypass. In: Buchwald's atlas of metabolic \& bariatric surgical techniques and procedures. Minnesota: Elsevier; 2012. pp. 114-41.

50. Sjöström L, Narbro K, Sjöström CD, Karason K, Larsson B, Wedel H, et al. Effects of bariatric surgery on mortality in swedish obese subjects. N Engl J Med. 2007:357:741-52.

51. Sjöström L1, Lindroos AK, Peltonen M, Torgerson J, Bouchard C, Carlsson B, Swedish Obese Subjects Study Scientific Group, et al. Lifestyle, diabetes, and cardiovascular risk factors 10 years after bariatric surgery. N Engl J Med. 2004;351:2683-93.

52. Golzarand M, Toolabi K, Farid R. The bariatric surgery and weight losing: a meta-analysis in the long- and very long-term effects of laparoscopic adjustable gastric banding, laparoscopic Roux-en-Y gastric bypass and laparoscopic sleeve gastrectomy on weight loss in adults. Surg Endosc. 2017;31:4331-45.

53. Welbourn R, Hollyman M, Kinsman R, Dixon J, Liem R, Ottosson J, et al. Bariatric surgery worldwide: baseline demographic description and one-year outcomes from the fourth ifso global registry report 2018. Obes Surg. 2019;29:782-95.

54. Angrisani L, Santonicola A, lovino P, Vitiello A, Higa K, Himpens J, et al. IFSO worldwide survey 2016: primary, endoluminal, and revisional procedures. Obes Surg. 2018;28:3783-94.

55. Buchwald H, Estok R, Fahrbach K, Banel D, Jensen MD, Pories WJ, et al. Weight and type 2 diabetes after bariatric surgery: systematic review and meta-analysis. Am J Med. 2009;122:248-56.

56. Herrera MF, García-García E, Arellano-Ramos JF, Madero MA, Aldrete-Velasco JA, Corvalá JAL. Metabolic surgery for the treatment of diabetes mellitus positioning of leading medical associations in Mexico. Obes Surg. 2018;28:3474-83.

57. Cancello R, Zulian A, Gentilini D, Mencarelli M, Della Barba A, Maffei M, et al. Permanence of molecular features of obesity in subcutaneous adipose tissue of ex-obese subjects. Int J Obes (Lond). 2013;37:867-73.

58. Aghamohammadzadeh R, Greenstein A, Yadav R, Jeziorska M, Hama S, Soltan $F$, et al. Effects of bariatric surgery on human small artery function. J Am Coll Cardiol. 2013;62:128-35.

59. Andersson DP, Hogling DE, Thorell A, Toft E, Qvisth V, Näslund E, et al. Changes in subcutaneous fat cell volume and insulin sensitivity after weight loss. Diabetes Care. 2014;37:1831-6.

60. Frikke-Schimdt H, Rourke RWO, Lumeng CN, Sandoval DA, Seeley RJ. Does bariatric surgery improve adipose tissue function? Obes Rev. 2016;17:795-809.

61. Evers SS, Sandoval DA, Seeley RJ. The physiology and molecular underpinnings of the effects of bariatric surgery on obesity and diabetes. Annu Rev Physiol. 2016;79:313-34.

62. Sandoval D. Bariatric surgeries: Beyond restriction and malabsorption Int J Obes (Lond). 2011;35:S45-9.

63. Stefater MA, Pérez-Tilve D, Chambers AP, Wilson-Pérez HE, Sandoval DA, Berger J, et al. Sleeve gastrectomy induces loss of weight and fat mass in obese rats, but does not affect leptin sensitivity. Gastroenterology. 2010;138:2426-36.

64. Grayson BE, Schneider KM, Woods SC, Seeley RJ. Improved rodent maternal metabolism but reduced intrauterine growth after vertical sleeve gastrectomy. Sci Transl Med. 2013;5:199ra112.

65. Odstrcil EA, Martinez JG, Santa Ana CA, Xue B, Schneider RE, Steffer KJ, et al. The contribution of malabsorption to the reduction in net energy absorption after long-limb Roux-en-Y gastric bypass. Am J Clin Nutr. 2010;92:704-13.

66. Bal BS, Finelli FC, Koch TR. Origins of and recognition of micronutrient deficiencies after gastric bypass surgery. Curr Diab Rep. 2011;11:136-41.

67. Ullrich J, Ernst B, Wilms B, Thurnheer M, Schultes B. Roux-en Y gastric bypass surgery reduces hedonic hunger and improves dietary habits in severely obese subjects. Obes Surg. 2013;23:50-5.

68. Cushing CC, Benoit SC, Peugh JL, Reiter-Purtill J, Inge TH, Zeller MH. Longitudinal trends in hedonic hunger after Roux-en-Y gastric bypass in adolescents. Surg Obes Relat Dis. 2014;10:125-30.

69. Wilson-Pérez HE, Chambers AP, Sandoval DA, Stefater MA, Woods SC Benoit SC, et al. The effect of vertical sleeve gastrectomy on food choice in rats. Int J Obes (Lond). 2013;37:288-95.

70. Ochner CN, Kwok Y, Conceição E, Pantazatos SP, Puma LM, Carnell S, et al. Selective reduction in neural responses to high calorie foods following gastric bypass surgery. Ann Surg. 2011;253:502-7.

71. Aron-Wisnewsky J, Clement K. The effects of gastrointestinal surgery on gut microbiota: potential contribution to improved insulin sensitivity. Curr Atheroscler Rep. 2014;16:454.

72. Ryan K, Tremaroli V, Clemmensen C, Kovatcheva-Datchary P, Myronovych $A$, Karns $R$, et al. FXR is a molecular target for the effects of vertical sleeve gastrectomy. Nature. 2014:509:183-8.

73. Liou AP, Paziuk M, Luevano J-M, Machineni S, Turnbaugh PJ, Kaplan LM. Conserved shifts in the gut microbiota due to gastric bypass reduce host weight and adiposity. Sci Transl Med. 2013;5:178ra41. 
74. Spinelli V, Lalloyer F, Baud G, Osto E, Kouach M, Daoudi M, et al. Influence of Roux-en-Y gastric bypass on plasma bile acid profiles: a comparative study between rats, pigs and humans. Int J Obes (Lond). 2016;40:1260-7.

75. Myronovych A, Kirby M, Ryan KK, Zhang W, Jha P, Setchell KD, et al. Vertical sleeve gastrectomy reduces hepatic steatosis while increasing serum bile acids in a weight-loss-independent manner. Obesity (Silve Spring). 2014;22:390-400

76. Hylemon PB, Zhou H, Pandak WM, Ren S, Gil G, Dent P. Bile acids as regulatory molecules. J Lipid Res. 2009;50:1509-20.

77. Thomas C, Auwerx J, Schoonjans K. bile acids and the membrane bile acid receptor TGR5-connecting nutrition and metabolism. Thyroid. 2008; 18:167-74.

78. Ryan KK, Kohli R, Gutierrez-Aguilar R, Gaitonde SG, Woods SC Seeley RJ. Fibroblast growth factor-19 action in the brain reduces food intake and body weight and improves glucose tolerance in male rats. Endocrinology. 2013;154:9-15.

79. Chambers AP, Jessen L, Ryan KK, Sisley S, Wilson-Pérez HE Stefater MA, et al. Weight-independent changes in blood glucose homeostasis after gastric bypass or vertical sleeve gastrectomy in rats. Gastroenterology. 2011;141:950-8.

80. Chambers AP, Stefater MA, Wilson-Perez HE, Jessen L, Sisley S, Ryan KK et al. Similar effects of roux-en-Y gastric bypass and vertical sleeve gastrectomy on glucose regulation in rats. Physiol Behav. 2011;105:120-3.

81. Rizzello M, Abbatini F, Casella G, Alessandri G, Fantini A, Leonetti F, et al. Early postoperative insulin-resistance changes after sleeve gastrectomy. Obes Surg. 2010;20:50-5.

82. Manning S, Pucci A, Batterham RL. GLP-1: a mediator of the beneficial metabolic effects of bariatric surgery? Physiology (Bethesda). 2015;30:50-62

83. Jørgensen NB, Dirksen C, Bojsen-Møller KN, Jacobsen SH, Worm D, Hansen DL, et al. Exaggerated glucagon-like peptide 1 response is important for improved $\beta$-cell function and glucose tolerance after roux-en- $y$ gastric bypass in patients with type 2 diabetes. Diabetes. 2013:62:3044-52

84. Holst JJ. Postprandial insulin secretion after gastric bypass surgery: The role of glucagon-like peptide 1. Diabetes. 2011;60:2203-5.

85. Ye J, Hao Z, Mumphrey MB, Townsend RL, Patterson LM, Stylopoulos N et al. GLP-1 receptor signaling is not required for reduced body weight after RYGB in rodents. Am J Physiol Integr Comp Physiol. 2014;306:R352-62.

86. Wilson-Perez HE, Chambers AP, Ryan KK, Li B, Sandoval DA, Stoffers D, et al. Vertical sleeve gastrectomy is effective in two genetic mouse models of glucagon-like Peptide 1 receptor deficiency. Diabetes. 2013; 62:2380-5.
87. Nannipieri M, Mari A, Anselmino M, Baldi S, Barsotti E, Guarino D, et al. The role of $\beta$-cell function and insulin sensitivity in the remission of type 2 diabetes after gastric bypass surgery. J Clin Endocrinol Metab. 2011; 96:E1372-9.

88. Romero F, Nicolau J, Flores L, Casamitjana R, Ibarzabal A, Lacy A, et al. Comparable early changes in gastrointestinal hormones after sleeve gastrectomy and Roux-En-Y gastric bypass surgery for morbidly obese type 2 diabetic subjects. Surg Endosc. 2012;26:2231-9.

89. Chambers AP, Smith EP, Begg DP, Grayson BE, Sisley S, Greer T, et al. Regulation of gastric emptying rate and its role in nutrient-induced GLP-1 secretion in rats after vertical sleeve gastrectomy. Am J Physiol Metab. 2014;306:E424-32.

90. Abbatini F, Rizzello M, Casella G, Alessandri G, Capoccia D, Leonetti F, et al. Long-term effects of laparoscopic sleeve gastrectomy, gastric bypass, and adjustable gastric banding on type 2 diabetes. Surg Endosc. 2010;24:1005-10.

91. Magkos F, Bradley D, Schweitzer GG, Finck BN, Eagon JC, Ilkayeva O, et al. Effect of Roux-en-Y gastric bypass and laparoscopic adjustable gastric banding on branched-chain amino acid metabolism. Diabetes. 2013;62:2757-61.

92. Dirksen C, Jørgensen NB, Bojsen-Møller KN, Jacobsen SH, Hansen DL Worm $\mathrm{D}$, et al. Mechanisms of improved glycaemic control after Rouxen-Y gastric bypass. Diabetologia. 2012;55:1890-901.

93. Schwartz MW, Woods SC, Porte D, Seeley RJ, Baskin DG. Central nervous system control of food intake. Nature. 2000;404:661-71.

94. Tao YX. The melanocortin-4 receptor: physiology, pharmacology, and pathophysiology. Endocr Rev. 2010;31:506-43.

95. Faroogi IS, Yeo GSH, Keogh JM, Aminian S, Jebb SA, Butler G, et al Dominant and recessive inheritance of morbid obesity associated with melanocortin 4 receptor deficiency. J Clin Invest. 2000;106:271-9.

96. Vaisse C, Clement K, Durand E, Hercberg S, Guy-Grand B, Froguel P. Melanocortin-4 receptor mutations are a frequent and heterogeneous cause of morbid obesity. J Clin Invest. 2000;106:253-62.

97. Mul JD, Begg DP, Alsters SIM, Haaften G van, Duran KJ, D'Alessio DA, et al. Effect of vertical sleeve gastrectomy in melanocortin receptor 4-deficient rats. Am J Physiol Endocrinol Metab. 2012;303:E103-10.

98. Müller TD, Nogueiras R, Andermann ML, Andrews ZB, Anker SD, Argente J, et al. Ghrelin. Mol Metab. 2015;4:437-60.

99. Chambers AP, Kirchner $\mathrm{H}$, Wilson-Perez HE, Willency JA, Hale JE, Gaylinn BD, et al. The effects of vertical sleeve gastrectomy in rodents are ghrelin independent. Gastroenterology. 2013;144:50-2. 